\title{
On Recent Advances in Divisor Cordial Labeling of Graphs
}

\author{
Vishally Sharma, A. Parthiban* \\ Department of Mathematics, Lovely Professional University, Punjab, India
}

\begin{abstract}
Cite This Paper in the following Citation Styles
(a): [1] Vishally Sharma, A. Parthiban, "On Recent Advances in Divisor Cordial Labeling of Graphs," Mathematics and Statistics, Vol.10, No.1, pp. 140-144, 2022. DOI: 10.13189/ms.2022.100111

(b): Vishally Sharma, A. Parthiban, (2022). On Recent Advances in Divisor Cordial Labeling of Graphs. Mathematics and Statistics, 10(1), 140-144. DOI: 10.13189/ms.2022.100111
\end{abstract}

Copyright $@ 2022$ by authors, all rights reserved. Authors agree that this article remains permanently open access under the terms of the Creative Commons Attribution License 4.0 International License

\begin{abstract}
An assignment of intergers to the vertices of a graph $\bar{G}$ subject to certain constraints is called a vertex labeling of $\bar{G}$. Different types of graph labeling techniques are used in the field of coding theory, cryptography, radar, missile guidance, $x$-ray crystallography etc. A DCL of $\bar{G}$ is a bijective function $\bar{f}$ from node set $\bar{V}$ of $\bar{G}$ to $\{1,2,3, \ldots,|\bar{V}|\}$ such that for each edge $r s$, we allot 1 if $\bar{f}(r)$ divides $\bar{f}(s)$ or $\bar{f}(s)$ divides $\bar{f}(r) \& 0$ otherwise, then the absolute difference between the number of edges having $1 \&$ the number of edges having 0 do not exceed 1 , i.e., $\left|e_{\bar{f}}(0)-e_{\bar{f}}(1)\right| \leq 1$. If $\bar{G}$ permits a DCL, then it is called a DCG. A complete graph $K_{n}$, is a graph on $n$ nodes in which any 2 nodes are adjacent and lilly graph $I_{n}$ is formed by $2 K_{1, n}$ joining $2 P_{n}, n \geq 2$ sharing a common node. i.e., $I_{n}=2 K_{1, n}+2 P_{n}$, where $\bar{K}_{1, n}$ is a complete bipartite graph $\& P_{n}$ is a path on $n$ nodes. In this paper, we propose an interesting conjecture concerning DCL for a given $\bar{G}$, besides, discussing certain general results concerning DCL of complete graph $K_{n}$-related graphs. We also prove that $I_{n}$ admits a DCL for all $n \geq 2$. Further, we establish the DCL of some $I_{n}$-related graphs in the context of some graph operations such as duplication of a node by an edge, node by a node, extension of a node by a node, switching of a node, degree splitting graph, \& barycentric subdivision of the given $\bar{G}$.
\end{abstract}

Keywords Graph Labeling, DCL, Lilly Graph

\section{Introduction}

By $\bar{G}$, we denote a simple, finite, \& undirected graph with node set $\bar{V} \&$ edge set $\bar{E}$. An allocation of labels to nodes or edges or sometimes both, under some constraints is called as graph labeling. Graph labeling is a close association of graph theory \& number theory. Being interdisciplinary, graph labeling is attracting the attention of numerous researchers and software developers. For number theory and graph theory related terms, we refer to [1] and [4], respectively. For further study on various graph labeling problems, see [3]. We use DCL and DCG to denote divisor cordial labeling and divisor cordial graph, respectively.

Cahit [2] introduced the idea of cordial labeling. Sundaram et al. [9] coined the notion of prime cordial labeling. The concept of DCL was given by Vartharajan et al. [10]. Vartharajan et al. [11] proved some general results especially the DCL of full binary tree.

Definition 1. [10] A DCL of $\bar{G}$ having $\bar{V}$ is a bijection $\bar{f}$ from $\bar{V}$ to $\{1,2,3, \ldots,|\bar{V}|\}$ such that each edge $r s$ is alloted 1 if $\bar{f}(r) / \bar{f}(s)$ or $\bar{f}(s) / \bar{f}(r) \& 0$ otherwise, then $\left|e_{\bar{f}}(0)-e_{\bar{f}}(1)\right| \leq$ 1. If $\bar{G}$ admits a DCL, then it is said to be a DCG.

For further results on DCL, refer to $[3,6,10,11]$.

\section{Main Results}

This section is devoted to derive some general results on DCG. Also, DCL of lilly graph in the context of different graph operations has been explored.

\subsection{DCL of $K_{n}$ Related Graphs}

Let $N(u)$ and $N[u]$ represent the open and closed neighbourhood of $u$, respectively. In this section, we deal with $K_{n}$ 
related graphs in establishing DCL.

Definition 2. [6] Let $\bar{G}$ with $V(\bar{G})=\bar{S}_{1} \cup \bar{S}_{2}, \ldots \cup \bar{S}_{t} \cup \bar{T}$, where each $\bar{S}_{i}$ is a set of nodes having at least two nodes of same degree \& $\bar{T}=V-\bigcup \bar{S}_{i}$. $D S(\bar{G})$ is the degree splitting graph of $\bar{G}$, which is created from $\bar{G}$ by adding nodes $\bar{w}_{1}, \bar{w}_{2}, \ldots, \bar{w}_{t} \&$ connecting $\bar{w}_{i}$ to each node of $\bar{S}_{i} ; 1 \leq i \leq t$.

Lemma 1. [7] $D S\left(K_{n}\right)$ yields $K_{n+1}$.

Theorem 1. [10] $K_{n}$ does not admit a DCL for $n \geq 7$.

Theorem 2. $D S\left(K_{n}\right)$ does not permit a DCL for $n \geq 6$.

Proof. The proof follows clearly from Theorem 1 and Lemma 1.

Definition 3. [5] Extension of a node $u_{i}^{\alpha}$, is achieved by adding a new node $u_{i}^{\prime}$ such that $N\left(u_{i}^{\prime}\right)=N\left[u_{i}^{\alpha}\right]$.

Lemma 2. Extension of any arbitrary node of $K_{n}$ yields $K_{n+1}$.

The proof follows from the fact that the newly added node is joined with all the nodes of $K_{n}$ including the node itself as every pair of nodes in $K_{n}$ are adjacent, which eventually gives rise to $K_{n+1}$.

Theorem 3. The graph $G$ obtained by performing extension of any arbitrary node in $K_{n}$ does not admit a DCL for $n \geq 6$.

Proof. It follows from Theorem 1 and Lemma 2.

Definition 4. [6] Switching of a node $v_{1}$ in $H_{1}$ is obtained by eliminating all edges incident to $v_{1}$ in $H_{1} \&$ adding new edges joining $v_{1}$ to every other node which were not adjacent to $v_{1}$ in $H_{1}$.

Lemma 3. The graph formed by switching any arbitrary node in $K_{n}$ admits a DCL for $n \leq 8$.

Proof. Switching of any arbitary node in $K_{n}$ results in a disconnected graph whose components are $K_{n-1}$ and $K_{1}$. The result clearly follows for switching of node in $K_{n}$ for $n=3,4,6,7$ (see Figure 1). Now we discuss DCL of switching of a node in $K_{5}$ and $K_{8}$.

Case(i). When $n=5$. Label the isolated node with 4 and assign the remaining labels to the nodes of $K_{4}$. Clearly, $e(0)=e(1)=3$.

Case(ii). When $n=8$. Label the isolated node with 7 and assign the remaining labels to the nodes of $K_{7}$. Here $e(0)=10$ and $e(1)=11$.

Theorem 4. Switching of an arbitrary node in $K_{n}$ for $n \geq 9$ does not admit a DCL.

Proof. Switching of an arbitrary node in $K_{n} ; n \geq 9$ results in a disconnected graph $G$ whose components are $K_{n-1}$ and $K_{1}$. We take $n=9$ for the sake of discussion. We obtain a disconnected $G$ obtained by switching of a node in $K_{9}$ whose

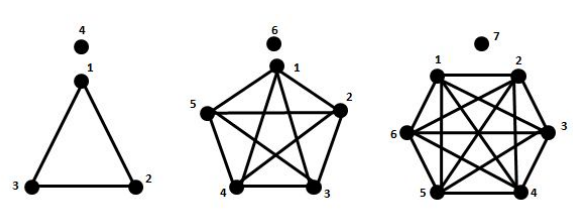

Figure 1. DCL of switching of a node in $K_{4}, K_{6}$ and $K_{7}$

componenets are $K_{8}$ and $K_{1}$. We prove by a method of contradiction. We assume that $G$ admits a DCL. Without loss of generality, label the isolated node with the largest prime $p$ where $p \leq 9$ (i.e., 7) in order to get more edges having label 1 , and assign the remaining labels to nodes of $K_{8}$ in any order. Here $e(0)=15, e(1)=13$, and therefore $|e(0)-e(1)|>1$, a contradiction. The other possibilities of assigning different labels to the isolated node can be dealt in the similar lines. The similar argument holds good for $n \geq 10$. Hence the theorem.

Considering the fact that characterization of DCGs is challenging in general, we propose the following conjecture.

Conjecture 1. For a given finite graph $\bar{G}$, establishing a $D C L$ of $\bar{G}$ is NP-hard.

Remark 1. We believe that the conjecture is true as there are no algorithm available in the literature and devising a particular pattern of DCG is also the hardest.

\subsection{DCL of Lilly Related Graphs}

Here, we explore the DCL of lilly graph and its related graphs in the context of various graph operations.

Definition 5. [8] Lilly graph $I_{n}$ is defined by $I_{n}=2 K_{1, n}+$ $2 P_{n}$ (see Figure 2).

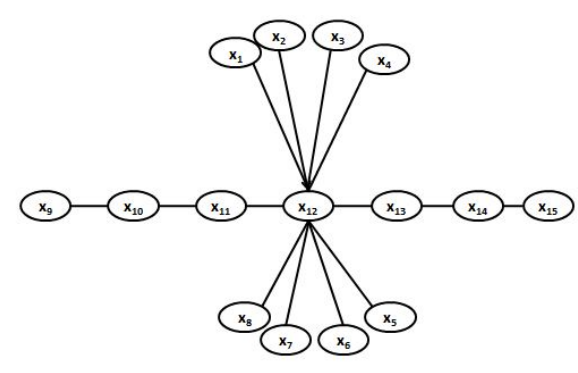

Figure 2. $I_{4}$

Theorem 5. $I_{n}$ admits a DCL.

Proof. Let $x_{1}, x_{2}, \ldots, x_{4 n-1}$ represent the nodes of $I_{n}$. Clearly, the number of elements in node \& edge sets are equal to $4 n-1$ and $4 n-2$, respectively. Define a function (bijective) $T: V\left(I_{n}\right) \rightarrow\{1,2, \ldots, 4 n-1\}$ by letting $T\left(x_{3 n}\right)=2$, $T\left(x_{1}\right)=4, T\left(x_{i}\right)=T\left(x_{i-1}\right)+2 ; 2 \leq i \leq 2 n-2$, $T\left(x_{2 n-1}\right)=1, T\left(x_{2 n-1+i}\right)=T\left(x_{2 n-1+i-1}\right)+2 ; 1 \leq$ $i<n+1, T\left(x_{3 n+1}\right)=T\left(x_{3 n-1}\right)+2, T\left(x_{3 n+1+i}\right)=$ $T\left(x_{3 n+1+(i-1)}\right)+2 ; 1 \leq i \leq n-2$. See that $e_{T}(0)=$ 
$e_{T}(1)=2 n-1$ which establishes that $I_{n}$ is a DCG (see Figure 3).

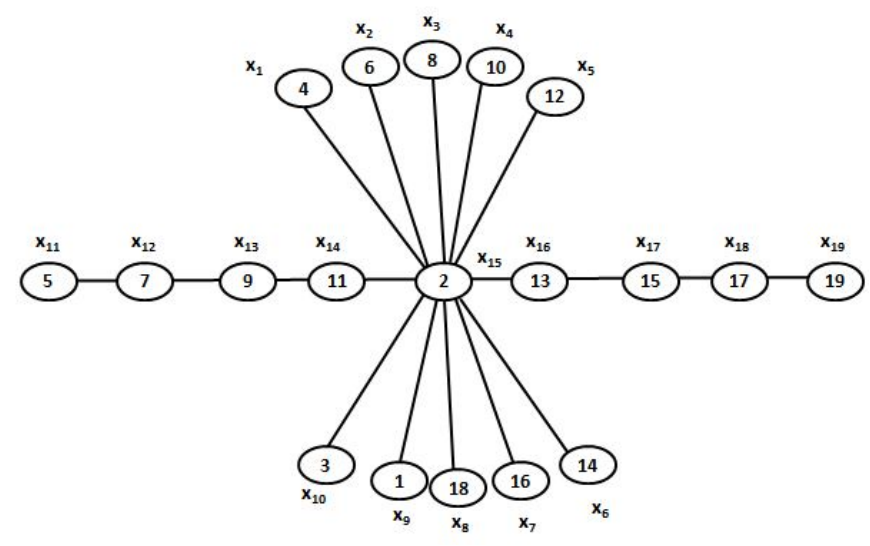

Figure 3. DCL of $I_{5}$

Theorem 6. Switching of any pendant node in $I_{n}$ admits a DCL.

Proof. Let $x_{1}, x_{2}, \ldots, x_{4 n-1}$ represent the nodes of $I_{n}$. Here, $x_{1}, x_{2}, \ldots, x_{n}, x_{n+1}, \ldots, x_{2 n}, x_{2 n+1}, x_{4 n-1}$ represent the pendant nodes. Let $\bar{G}$ be the newly constructed graph by switching any pendant node of $I_{n}$, say $x_{k}$. Clearly, the number of elements in node \& edge sets of $\bar{G}$ ) are equal to $4 n-1 \&$ $8 n-6$, respectively. Define a function (bijective) $T: \bar{V}(\bar{G}) \rightarrow$ $\{1,2, \ldots, 4 n-1\}$ by assigning $T\left(x_{k}\right)=1 \& T\left(x_{3 n}\right)=p$ where $p$ is the largest prime $\leq 4 n-1$. Label the rest of the nodes by using unutilized labels out of $\{1,2, \ldots, 4 n-1\}$. One can see that $4 n-3$ edges recieve the label 1 as $T\left(x_{k}\right)=1 \&$ there are exactly $4 n-3$ edges whose one end node is $x_{k}$. Consequently, the remaining edges get the label 0 . So $\left|e_{T}(0)-e_{T}(1)\right| \leq 1$ which shows that $\bar{G}$ is a DCG.

Corollary 7. Switching of any node of degree 2 in $I_{n}$ admits a DCL.

Proof. Switching a node of degree 2 in $I_{n}$ results in a graph having $4 n-1$ nodes and $8 n-8$ edges. The labeling is done on the similar lines as in Theorem 6.

Theorem 8. Switching of the apex node in $I_{n}$ admits a DCL.

Proof. Let $\bar{G}$ be obtained by switching the apex node of $I_{n}$, namely, $x_{3 n}$. Clearly, the number of elements in node \& edge sets of $\bar{G}$ are equal to $4 n-1 \& 4 n-8$, respectively. Define a function $T: \bar{V}(\bar{G}) \rightarrow\{1,2, \ldots, 4 n-1\}$ by assigning $T\left(x_{3 n}\right)=$ $1, T\left(x_{2 n+1}\right)=4, T\left(x_{i}\right)=T\left(x_{i-1}\right)+2 ; 2 n+2 \leq i \leq 3 n-1$, $T\left(x_{3 n+1}\right)=T\left(x_{3 n-1}\right)+2, T\left(x_{i}\right)=T\left(x_{i-1}\right)+2 ; 3 n+2 \leq$ $i \leq 4 n-1$. Now assigning the unutilized labels to unlabeled nodes in any order yields that $\left|e_{T}(0)-e_{T}(1)\right| \leq 1$ (see Figure 4).

Definition 6. [6] Duplication of a node $z_{i}$ with a node $z_{i}{ }^{\prime}$ results in $\bar{G}$ having the property that $N\left(z_{i}\right)=N\left(z_{i}{ }^{\prime}\right)$.
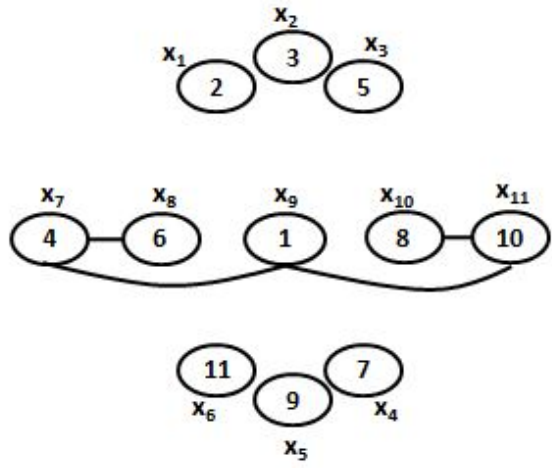

Figure 4. DCL of switching of $x_{3 n}$ in $I_{3}$

Theorem 9. Duplication of the apex node in $I_{n}$ admits a DCL.

Proof. Let $\bar{G}$ be formed by duplicating the apex node $x_{3 n}$ of $I_{n}$ by the newly added node $s$. Clearly, the number of elements in node \& edge sets of $\bar{G}$ are given by $4 n$ and $6 n$, respectively. Define a function $T: \bar{V}(\bar{G}) \rightarrow\{1,2, \ldots, 4 n\}$ by assigning $T\left(x_{3 n}\right)=2, T(s)=4, T\left(x_{1}\right)=1, T\left(x_{2}\right)=6, T\left(x_{i}\right)=$ $T\left(x_{i-1}\right)+2 ; 3 \leq i \leq 2 n-1, T\left(x_{2 n}\right)=5, T\left(x_{2 n+1}\right)=3$ and $T\left(x_{2 n+2}\right)=9$. Now allocate the unutilized labels from $\{1,2, \ldots, 4 n\}$ simultaneously to the unlabeled nodes $x_{j} ; 2 n+$ $3 \leq j \leq 4 n-1$ and $j \neq 3 n$. It can be easily seen that $\left|e_{T}(0)-e_{T}(1)\right| \leq 1$ which shows that $\bar{G}$ is a DCG.

Theorem 10. The duplication of an arbitrary node of degree 1 or 2 in $I_{n}$ permits a DCL.

Proof. Let $\bar{G}$ be formed by duplicating any arbitrary node $x_{k}$ of $I_{n}$ by the the newly inserted node $s$. Then the cardinality of node set of $\bar{G}$ is $4 n$. Now arise two cases.

Case(i). Duplication of a node of degree 1.

In this case, the cardinality of edge set of $\bar{G}$ is $4 n-1$. Define a function (bijective) $T: \bar{V}(\bar{G}) \rightarrow\{1,2, \ldots, 4 n\}$ by fixing $T\left(x_{3 n}\right)=2, T\left(x_{1}\right)=1$ and $T(s)$ be the largest prime $p \leq 4 n$. Assign the unutilized even labels to $x_{i} ; 2 \leq i \leq 2 n$ and odd labels simultaneously to $x_{j} ; 2 n+1 \leq j \leq 4 n-1, j \neq 3 n$.

Case(ii). Duplication of a node of degree 2 .

In this case, the cardinality of edge set of $\bar{G}$ is $4 n$. Labeling is done by using the pattern of case(i) (see Figure 5).

In both the cases, we observe that the difference of edges having labels 1 and 0 is not more than 1 which verifies that $\bar{G}$ is a DCG.

\section{Theorem 11. $D S\left(I_{n}\right)$ permits a DCL.}

Proof. Let $\bar{G}$ denote the $D S\left(I_{n}\right)$ with $\bar{V}(\bar{G})=V\left(I_{n}\right) \cup$ $\{v, w\}$ and $\bar{E}(\bar{G})=E\left(I_{n}\right) \cup\left\{x_{i} v: 1 \leq i \leq 2 n\right\} \cup$ $\left\{x_{2 n+1} v, x_{4 n-1} v\right\} \cup\left\{x_{i} w: 2 n+2 \leq i \leq 4 n-2, i \neq 3 n\right\}$. The number of elements in node \& edge sets of $\bar{G}$ are respectively equal to $4 n+1 \& 8 n-4$. Consider a bijective map $T: \bar{V}(\bar{G}) \rightarrow\{1,2, \ldots, 4 n+1\}$ determined by choosing $T\left(x_{3 n}\right)=1, T(v)=4, T(w)=2, T\left(x_{4 n-1}\right)=4 n-2$, $T\left(x_{1}\right)=3, T\left(x_{i}\right)=T\left(x_{i-1}\right)+2 ; 2 \leq i \leq 2 n$ and 


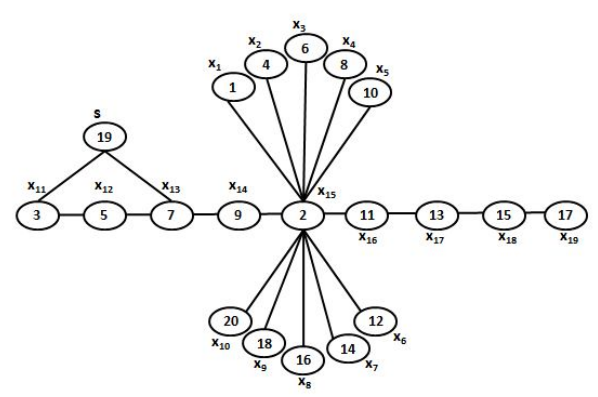

Figure 5. DCL of duplication of node $x_{12}$ of degree 2 in $I_{5}$

$T\left(x_{2 n+1}\right)=6$. Assign the unutilized labels to the remaining nodes in any order. It follows that $\bar{G}$ is a DCG (see Figure $6)$.

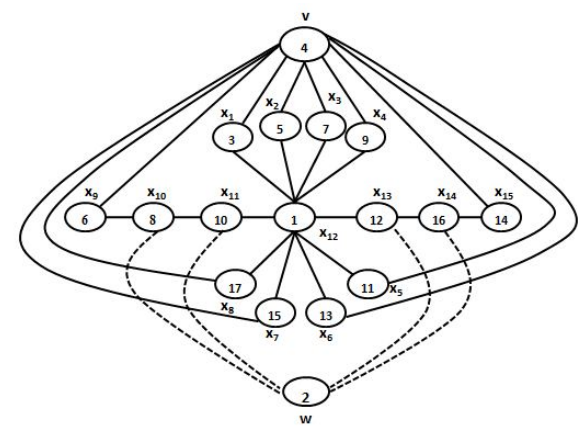

Figure 6. DCL of degree splitting graph of $I_{4}$

Definition 7. [6] Let $e=r s \in \bar{G}$. Then $e$ is subdivided once it is substituted by edges $e^{\prime}=r w \& e^{\prime \prime}=w s$. If all edges in $\bar{G}$ are subdivided, we obtain a graph which we call as barycentric subdivision of $\bar{G}$.

Theorem 12. Barycentric subdivision of $I_{n}$ permits a DCL.

Proof. Let $\bar{G}$ represent the barycentric subdivision of $I_{n}$. Here, $\bar{V}(\bar{G})=V\left(I_{n}\right) \cup\left\{v_{1}, v_{2}, \ldots, v_{2 n}, v_{2 n+1}, \ldots, v_{4 n-2}\right\} \&$ $\bar{E}(\bar{G})=\left\{x_{3 n} v_{i}: 1 \leq i \leq 2 n\right\} \cup\left\{v_{i} x_{i}: 1 \leq i \leq\right.$ $2 n\} \cup\left\{x_{i} v_{i}: 2 n+1 \leq i \leq 4 n-2\right\} \cup\left\{v_{i} x_{i+1}: 2 n+1 \leq\right.$ $i \leq 4 n-2\}$. Clearly, the number of elements in node \& edge sets of $\bar{G}$ are respectively equal to $8 n-3 \& 8 n-4$. Consider a function (bijective) $T: \bar{V}(\bar{G}) \rightarrow\{1,2, \ldots, 8 n-3\}$ by choosing $T\left(x_{3 n}\right)=2, T\left(x_{1}\right)=1, T\left(v_{1}\right)=4, T\left(v_{2}\right)=6$, $T\left(v_{i}\right)=T\left(v_{i-1}\right)+4 ; 3 \leq i \leq 2 n-1$,

$T\left(x_{i}\right)=\frac{T\left(v_{i}\right)}{2} ; 2 \leq i \leq 2 n-1$,

$T\left(v_{2 n}\right)=T\left(x_{2 n-1}\right)+2 \& T\left(x_{2 n}\right)=T\left(v_{2 n}\right)+2$.

Next, fix $T\left(x_{2 n+1}\right)=8, T\left(v_{2 n+1}\right)=12$,

$T\left(x_{i}\right)=T\left(x_{i-1}\right)+8 ; 2 n+2 \leq i \leq 3 n-1$,

$T\left(v_{i}\right)=T\left(v_{i-1}\right)+8 ; 2 n+2 \leq i \leq 3 n-2$,

$T\left(x_{4 n-1}\right)=T\left(x_{3 n-1}\right)+4, T\left(v_{3 n-1}\right)=T\left(x_{2 n}\right)+2$,

$T\left(v_{3 n}\right)=T\left(v_{3 n-1}\right)+2, T\left(x_{3 n+1}\right)=T\left(v_{3 n}\right)+2$,

$T\left(v_{i}\right)=T\left(v_{i-1}\right)+4 ; 3 n+1 \leq i \leq 4 n-2$

$T\left(x_{i}\right)=T\left(x_{i-1}\right)+4 ; 3 n+2 \leq i \leq 4 n-2$.

Evidently, $e_{T}(1)=4 n-2 \& e_{T}(0)=4 n-2$ which proves that $\bar{G}$ is DCG (see Figure 7).

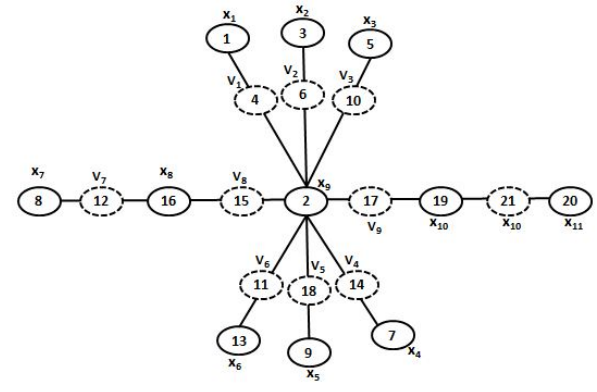

Figure 7. DCL of barycentric subdivision of $I_{3}$

Theorem 13. Extension of all pendant nodes in $I_{n}$ permits a DCL.

Proof. Let $\bar{G}$ be formed by performing the extension of all pendant nodes of $I_{n}$ with $\bar{V}(\bar{G})=$ $V\left(I_{n}\right) \cup\left\{v_{1}, v_{2}, \ldots, v_{2 n}, \quad v_{2 n+1}, v_{4 n-1}\right\}$ and $\bar{E}(\bar{G})=$ $E\left(I_{n}\right) \cup\left\{x_{i} v_{i} ; 1 \leq i \leq 2 n\right\} \cup\left\{v_{i} x_{3 n} ; 1 \leq i \leq\right.$ $2 n\} \cup\left\{x_{2 n+1} v_{2 n+1}, v_{2 n+1} x_{2 n+2}, x_{4 n-1} v_{4 n-1}, v_{4 n-1} x_{4 n-2}\right\}$. Clearly, the number of elements in node \& edge sets of $\bar{G}$ are given by $6 n+1 \& 8 n+2$ respectively. Consider a function $T: \bar{V}(\bar{G}) \rightarrow\{1,2, \ldots, 6 n+1\}$ defined by letting $T\left(x_{3 n}\right)=2$, $T\left(x_{1}\right)=1, T\left(x_{2}\right)=6, T\left(v_{1}\right)=4$. Now two cases arise.

Case 1 . when $n$ is even.

$T\left(x_{i}\right)=T\left(x_{i-1}\right)+4 ; 3 \leq i \leq n+\frac{n}{2}$,

$T\left(v_{i}\right)=\frac{T\left(x_{i}\right)}{2} ; 2 \leq i \leq n+\frac{n}{2}$,

$T\left(x_{n+\frac{n}{2}+1}\right)=8, T\left(x_{i}\right)=T\left(x_{i-1}\right)+8 ; n+\frac{n}{2}+2 \leq i \leq 2 n$, $T\left(v_{i}\right)=T\left(x_{i}\right)+4 ; n+\frac{n}{2}+1 \leq i \leq 2 n$, $T\left(v_{2 n+1}\right)=T\left(v_{n+\frac{n}{2}}\right)+2$ and $T\left(v_{4 n-1}\right)=6 n+1$.

Assign all the unutilized even labels and then odd labels to $x_{2 n+1}, x_{2 n+2}, \ldots, x_{4 n-2}$ (excluding $x_{3 n}$ ) simultaneously from $\{1,2, \ldots, 6 n+1\}$.

Case 2. When $n$ is odd.

$T\left(x_{i}\right)=T\left(x_{i-1}\right)+4 ; 3 \leq i \leq n+\left\lceil\frac{n}{2}\right\rceil$,

$T\left(v_{i}\right)=\frac{T\left(x_{i}\right)}{2} ; 2 \leq i \leq n+\left\lceil\frac{n}{2}\right\rceil$,

$T\left(x_{n+\left\lceil\frac{n}{2}\right\rceil+1}\right)=8, T\left(x_{i}\right)=T\left(x_{i-1}\right)+8 ; n+\left\lceil\frac{n}{2}\right\rceil+2 \leq i \leq$ $2 n$,

$T\left(v_{i}\right)=T\left(x_{i}\right)+4 ; n+\left\lceil\frac{n}{2}\right\rceil+1 \leq i \leq 2 n$, $T\left(v_{2 n+1}\right)=T\left(v_{n+\left\lceil\frac{n}{2}\right\rceil}\right)+2$ and $T\left(v_{4 n-1}\right)=6 n+1$.

Allocate all unutilized even labels $\&$ then odd labels to $x_{2 n+1}, x_{2 n+2}, \ldots, x_{4 n-2}$ (excluding $x_{3 n}$ ) simultaneously from $\{1,2, \ldots, 6 n+1\}$.

In both the cases $T$ induces DCL for $\bar{G}$.

Theorem 14. Extension of the apex node in $I_{n}$ permits a DCL.

Proof. Let $\bar{G}$ be constructed by taking the extension of the apex node of $I_{n}$. Clearly, $\bar{V}(\bar{G})=V\left(I_{n}\right) \cup\{w\} \& \bar{E}(\bar{G})=$ $E\left(I_{n}\right) \cup\left\{x_{i} w: 1 \leq i \leq 2 n\right\} \cup\left\{x_{3 n} w, x_{3 n-1} w, x_{3 n+1} w\right\}$. Note here, $|\bar{V}(\bar{G})|=4 n \&|\bar{E}(\bar{G})|=6 n+1$. Define a function (bijective) $T: \bar{V}(\bar{G}) \rightarrow\{1,2, \ldots, 4 n\}$ by choosing $T\left(x_{3 n}\right)=$ $2, T(w)=4, T\left(x_{1}\right)=6, T\left(x_{i}\right)=T\left(x_{i-1}\right)+2 ; 2 \leq i \leq 2 n-$ $2, T\left(x_{2 n-1}\right)=1$ and $T\left(x_{2 n}\right)=3$. Assign the unutilized odd labels simultaneously to $x_{2 n+1}, x_{2 n+2}, \ldots, x_{4 n-1}$. It follows 
that $\bar{G}$ is a DCG.

Conclusion: This paper has dealt with certain interesting general results on DCL for $K_{n}$ related graphs besides, formulating an impressive conjecture on DCL. Further, we have proved that lilly graph admits a DCL and discussed DCL for some lilly related graphs under various graph operations.

\section{REFERENCES}

[1] Burton David, "Elementary Number Theory", 2nd ed, Wm.C.Brown Company Publishers, 1980.

[2] Cahit I, "Cordial Graphs: A Weaker Version of Graceful and Harmonious Graphs, Ars Combinatoria, vol. 23, pp. 201-207, 1987.

[3] Gallian J. A, "A Dynamic Survey of Graph Labeling”, Electronic Journal of Combinatorics, vol. 16, DS6, 2009.

[4] Hararay F, "Graph Theory”, Addison Wesley Reading, 1972.

[5] Parthiban A and David N. G, "Prime Distance Labeling of Some Path Related Graphs", International Journal of Pure and Applied Mathematics, vol. 120, no. 7, pp. 5967, 2018.
[6] Parthiban A and Sharma V, "A Comprehensive Survey on Prime Cordial and Divisor Cordial Labeling of Graphs", Journal of Physics: Conference Series, vol. 1531, no. 012074, 2020. DOI: 10.1088/1742-6596/1531/1/012074.

[7] Ponraj R and Somasundaram S, "On the Degree Splitting Graph of a Graph", National Academy Science Letters, vol. 27 , no. 7 and 8, pp. 275-278, 2004

[8] Samuel Edward A and Kalaivani S, "Square Sum Labeling for Some Lilly Related Graphs", International Journal of Advanced Technology and Engineering Exploration, vol. 4, no. 29, pp. 68-72, 2017.

[9] Sundaram M, Ponraj R, Somasundaram S, "Prime Cordial Labeling of Graphs", Journal of Indian Academy of Mathematics, vol. 27, no. 2, pp. 373-390, 2005.

[10] Varatharajan R, Navanaeethakrishnan S, Nagarajan K, "Divisor Cordial Graphs", International Journal of Math. Combinatorics, vol. 4, pp. 15-25, 2011.

[11] Varatharajan R, Navanaeethakrishnan S, Nagarajan K, "Special Classes of Divisor Cordial Graphs", International Mathematical Forum, vol. 7, no. 35, pp. 17371749, 2012. 